\title{
Motivando el aprendizaje activo del Diseño de Experimentos en Ingeniería mediante actividades virtuales
}

\author{
V. Chirivella, R.M. Alcover, B. Richart \\ Departamento de Estadística e Investigación Operativa Aplicadas y Calidad \\ Universitat Politècnica de València \\ \{vchirive, ralcover, brichart\}@eio.upv.es
}

\section{Abstract}

In this work we analyze the use of a simulator on virtual laboratories as an alternative to a real laboratory in the subjects of Design of Experiments (DOE) on the university master's degree in Data Analysis Engineering, Process Improvement and Decision Making offered by the Polytechnic University of Valencia (UPV). This change is motivated by the restrictive conditions of use of the laboratories that have been imposed due to COVID-19, and the need to continue using active methodologies in these subjects. The use of simulators has turned out to be an adequate solution to circumvent those restrictions, while maintaining all the advantages of the current methodology, which covers a large number of competencies and improves student learning.

Keywords: Design of Experiments; Virtual Laboratory; Simulators; Educational innovation; Active methodologies; Soft skills; Active learning

\section{Resumen}

En este trabajo se analiza el uso de un simulador en un laboratorio virtual como alternativa a un laboratorio real en las asignaturas de Diseño de Experimentos (DOE) del máster universitario de Ingeniería de Análisis de Datos, Mejora de Procesos y Toma de Decisiones ofertado por la Universitat Politècnica de València (UPV). Este cambio viene motivado por las restrictivas condiciones de uso de los laboratorios que se han impuesto por causa del COVID-19, y por la necesidad de seguir utilizando metodologías activas en dichas asignaturas. El uso de simuladores ha resultado ser una solución adecuada para soslayar las restricciones impuestas a los espacios destinados a laboratorios, a la vez que se mantienen todas las ventajas de la metodología utilizada actualmente, la cual cubre un gran número de competencias y mejora el aprendizaje de los estudiantes.

Palabras clave: Diseño de Experimentos; Laboratorio virtual; Simulador; Innovación educativa; Metodologías activas; Competencias; Aprendizaje activo

\section{Introducción}

En el ámbito de la ingeniería y en un escenario internacional con mercados cada vez más competitivos en los que surgen continuamente nuevos productos y problemas que hay que solucionar, confiar en que la experiencia acumulada a lo largo de los años sea la única guía en la mejora de procesos, sistemas y productos, supone como mínimo, correr el riesgo de que la competencia tome la delantera y aumente sus cuotas de mercado a costa de los más rezagados. Por ello, para mejorar las características de los productos y de los procesos, los ingenieros deben recurrir de forma sistemática a la experimentación, base de todas las ciencias empíricas (Romero y Zúnica, 2004). La experimentación es el procedimiento que permite al ingeniero incrementar sus conocimientos de forma eficaz y eficiente. Así, cuando en el curso 2009-2010, la UPV puso en marcha el máster universitario en INGENIERÍA DE ANÁLISIS DE DATOS, MEJORA DE PROCESOS Y TOMA DE DECISIONES, se decidió que la materia de Diseño de Experimentos (DOE) 
debía ubicarse en el bloque de Mejora de Procesos de su plan de estudios, impartirse durante el primer semestre del curso y ser de carácter obligatorio para todos los alumnos matriculados.

La materia de DOE se compone de dos asignaturas, DOE I y DOE Avanzado, cada una de ellas con 3 créditos ECTS. En estas asignaturas se trabajan conceptos fundamentales de la metodología del diseño experimental, así como otros temas más avanzados tales como el Diseño Robusto de productos y procesos y el Diseño de Mezclas.

En un trabajo anterior (Chirivella, Alcover y Richart, 2018), se presentó el importante efecto positivo que había tenido en el aprendizaje de ambas asignaturas de máster la realización de actividades por parte del alumnado que motivan y promuevan su participación activa. En el trabajo mencionado se constata que, tras la modificación de la metodología docente tradicionalmente seguida en las asignaturas por otra más participativa y dinámica, y la introducción de una nueva actividad práctica, los resultados han sido significativamente mejores, además de poder trabajar con ello nuevas competencias que previamente se habían pasado por alto.

Sin embargo, la actual pandemia de COVID-19 y las restricciones impuestas por el PROTOCOLO DE LA UPV, concretamente en cuanto a los puestos de trabajo en los laboratorios (aforos restringidos, una persona por puesto y siempre con mascarilla, $5 \mathrm{~m}^{2}$ libres a su alrededor), ponía en peligro durante el curso 20202021 la realización de las actividades prácticas grupales, al menos tal y como estaban diseñadas hasta el momento en las asignaturas. Adicionalmente, el problema se complicaba todavía más en los casos de alumnos que, estando matriculados, no podían acceder físicamente al laboratorio por las restricciones de movilidad impuestas por los gobiernos nacional y autonómico. Y concretamente en nuestras asignaturas, no sólo restricciones a los alumnos situados en otras provincias de España, sino también a los situados en otros países, en especial a los que vienen habitualmente de América Central y del Sur. En el curso 20202021 los profesores de DOE debíamos combinar los conceptos de "aprendizaje activo", "actividad grupal" y "docencia no presencial síncrona".

La solución a este problema es la misma que la aportada a la teoría, convertir la actividad presencial en una actividad en línea, mediante la creación de un laboratorio virtual (Torres, 2004; Potkonjak, 2016). Los laboratorios virtuales son plataformas interactivas que permiten llevar a cabo actividades de laboratorio sin que el alumno tenga que cambiar de localización: en este caso su hogar (allá donde esté), o el aula de teoría. Además de ser una herramienta que permite fomentar el aprendizaje autónomo, con un buen diseño (Stahre, 2019) un laboratorio virtual puede proporcionar las mismas ventajas que el laboratorio físico en cuanto al trabajo en equipo. Aunque existen laboratorios virtuales estadísticos, éstos se limitan a cuestiones básicas que quedan muy atrás en el máster, siendo necesario recurrir a laboratorios de ingeniería propiamente dichos, en el ámbito de la producción industrial.

En el presente trabajo presentamos el diseño, utilización y resultados de un laboratorio virtual que ha permitido a los alumnos de las asignaturas de DOE durante el curso 2020-2021 aprender de forma activa, aplicando los contenidos (muchos de ellos de considerable nivel de complejidad) en un contexto lúdico o de juego, colaborando con sus compañeros, incrementando el grado de interactividad y trabajando las diferentes competencias, todo ello en condiciones de pandemia por COVID-19.

Finalmente, hay que señalar que la experiencia adquirida durante el presente curso servirá, en primer lugar, como banco de pruebas para una posible versión no presencial del mencionado máster, indispensable si continúan las restricciones por la pandemia, o si se decide ofrecer en un futuro próximo las asignaturas de $D O E$ como no presenciales en el marco de un MOOC, dada su especial relevancia en el ámbito de la Ingeniería y en el contexto de la Calidad. En segundo lugar, y a escala más reducida, el trabajo llevado a cabo durante el curso 2020-2021 permitirá valorar la viabilidad de la sustitución de los enunciados 
"tradicionales" de las prácticas de las asignaturas por simulaciones sencillas, proporcionando a los alumnos su propio conjunto de datos para resolver problemas que pudieran plantearse en las diferentes unidades didácticas del temario.

La estructura del presente trabajo es la siguiente: tras la Introducción, en el siguiente apartado se presentan los Objetivos de la experiencia que se ha llevado a cabo. En el apartado 2 se describe el experimento que han realizado los alumnos, así como los diferentes conceptos a tener en cuenta en su evaluación. En el apartado 3 se muestran los resultados obtenidos en las asignaturas de $D O E$ tras la incorporación de la nueva actividad y se comparan con los del curso anterior. Finalmente, el apartado de Conclusiones recoge las principales conclusiones que los profesores de las asignaturas de $D O E$ hemos obtenido con la experiencia durante el curso 2020-2021.

\section{Objetivos}

La sustitución del experimento utilizado habitualmente como forma de evaluación de la asignatura se ha realizado atendiendo a los siguientes objetivos:

- Trabajar con los alumnos de forma no presencial síncrona utilizando metodologías activas.

- Valorar el uso de simuladores como forma sencilla de obtener los datos para efectuar su análisis en las sesiones de práctica de laboratorio.

- Seleccionar materiales adecuados para llevar a cabo un experimento simulado de un proceso industrial, o de un problema en el ámbito de la ingeniería.

- Elaborar una guía detallada para que el alumno pueda manejar el simulador, organizar y planificar su trabajo y gestionar el tiempo disponible para cada actividad.

- Motivar e involucrar de lleno al alumno en el análisis de un proceso, desde el planteamiento inicial hasta la presentación y la discusión de resultados.

- Trabajar en un equipo multidisciplinar.

- Mejorar el aprendizaje y con ello el rendimiento de los alumnos.

\section{Experiencia propuesta: medios e instrumentos de evaluación}

Teniendo en cuenta los objetivos propuestos, se ha realizado una selección de simuladores de libre acceso encontrados en internet para conformar nuestro laboratorio virtual, valorando el posible interés que pudiera despertar en nuestros alumnos y la facilidad de modificar su código para incluir elementos estocásticos que lo convierta en un simulador real, y no en un mero calculador. Algunos de los calculadores/simuladores que consideramos para el trabajo son:

- Biorreactor. Calcula la cantidad y concentración de biomasa que se consigue en un reactor de tanque agitado continuo, o reactor quimiostato.

- Catapulta trebuchet. Calcula la altura y distancia recorrida por un proyectil lanzado por una catapulta de tipo trebuquete.

- Cruce regulado por semáforos. Simulador de densidad de tráfico en un cruce regulado por semáforos y su comparación con una rotonda equivalente.

- Motor de iones. Calcula la fuerza y la duración del encendido de un motor de iones utilizado para situar en órbita solar a una sonda exploradora.

De todos los calculadores y simuladores considerados, se ha escogido el diseño y optimización de una catapulta Trebuchet porque es el proceso más sencillo de entender por alumnos sin conocimientos de ingeniería, y el programa base es muy visual e intuitivo. La aleatorización de los resultados se consigue 
asignando valores al azar en las pérdidas por rozamiento en el mecanismo, que se consideraban fijas inicialmente.

\section{El experimento de la catapulta}

Se desea optimizar el diseño una catapulta tipo Trebuchet (también llamado trebuquete o fundíbulo) de brazo flotante, como el que se presenta en la Figura 1, de tamaño "sobremesa" (40 cm de alto), de forma que arroje un proyectil lo más lejos posible a partir de la selección de ciertos factores de diseño y de uso.

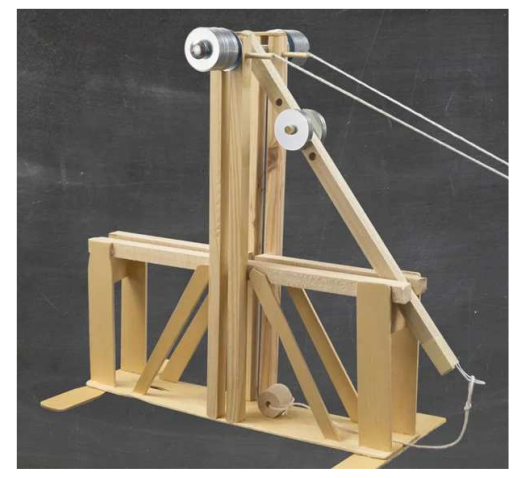

Figura 1 Trebuquete de brazo flotante (https://www.instructables.com/The-Floating-Arm-Trebuchet/)

Un FUNDÍBULO es un arma de asedio medieval, una catapulta de precisión que se utilizaba para destruir murallas o lanzar objetos por encima de las mismas. Esta precisión se debe a que utiliza la energía potencial de una gran masa (contrapeso) que se transfiere con poca variabilidad en energía cinética, lo que permite impulsar un proyectil siguiendo una trayectoria parabólica. Entre las mejoras del diseño original, se incluye la sustitución de un brazo oscilante en un eje, a un brazo flotante que gira y se desliza sobre dos ejes. El proyectil del fundíbulo solía ser una piedra grande o una esfera de piedra, aunque en ocasiones se empleaban otro tipo de proyectiles: animales muertos, colmenas, cabezas de enemigos decapitados, pequeñas esferas de arcilla cocida que explotarían al impacto como metralla, barriles de brea o aceite encendidos, o hasta negociadores que no habían tenido éxito, heces de animales, prisioneros de guerra o espías vivos, según Wikipedia.

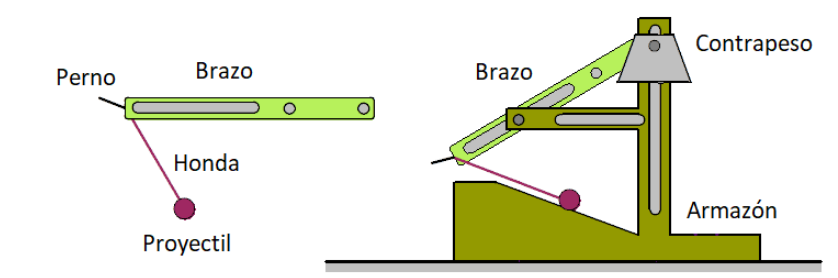

Figura 2 Trebuquete de brazo flotante (Eric Constans and Rowan University)

El trebuquete de brazo flotante consta de un armazón, compuesto de una viga central vertical con una canaladura que permite guiar la bajada en vertical de un contrapeso. Una segunda viga en horizontal, formando un ángulo recto, y unida a cierta altura con la primera, sirve de soporte por el que gira y se desliza una tercera viga, que hace de brazo lanzador. De este brazo cuelga en un extremo el contrapeso, y del otro una honda que contiene y permite lanzar los proyectiles, y en su centro existen unas ruedas que apoyan y se desplazan sobre la segunda viga. La honda está sujeta por una de las cuerdas al extremo del brazo, y la otra cuerda se engancha (sin atar) en un perno de ese mismo extremo del brazo.

Cuando se libera el contrapeso, éste baja de forma vertical moviendo un extremo del brazo hacia abajo. El otro extremo del brazo se apoya en la segunda viga, que hace de eje de giro. Cuando el brazo llega a la horizontal, sus ruedas se apoyan en la segunda viga y se convierten en un nuevo eje de giro. La honda se

(cc) BY-NC-ND 2021, Universitat Politècnica de València 
desplaza y gira con este movimiento, atada al extremo del brazo. En algún momento de la bajada del contrapeso la honda suelta al proyectil al liberarse del perno, y éste sale disparado hacia su objetivo. Un vídeo de la catapulta en acción se encuentra en FLOATING ARM TREBUCHET LAUNCH.

Ciertos factores de diseño de la catapulta derivan del hecho de su tamaño, y del material idealmente empleado, la madera. Así, una catapulta de $40 \mathrm{~cm}$ de alto no puede estar compuesta por barras de madera de una longitud o de un grosos inapropiados, por lo que se fijan sus dimensiones a ciertos valores razonables, que pueden encontrarse en la Tabla 1 y que están representados en la Figura 3.

Tabla 1 Dimensiones del trebuquete

\begin{tabular}{|l|l|}
\hline Factor & Valor $(\mathrm{m})$ \\
\hline $\mathrm{D}$ & 0,15 \\
\hline $\mathrm{w}$ & 0,04 \\
\hline $\mathrm{t}$ & 0,01 \\
\hline $\mathrm{W}$ & 0,2 \\
\hline $\mathrm{H}$ & 0,2 \\
\hline h0 & 0,4 \\
\hline$\lambda$ & 0 \\
\hline
\end{tabular}

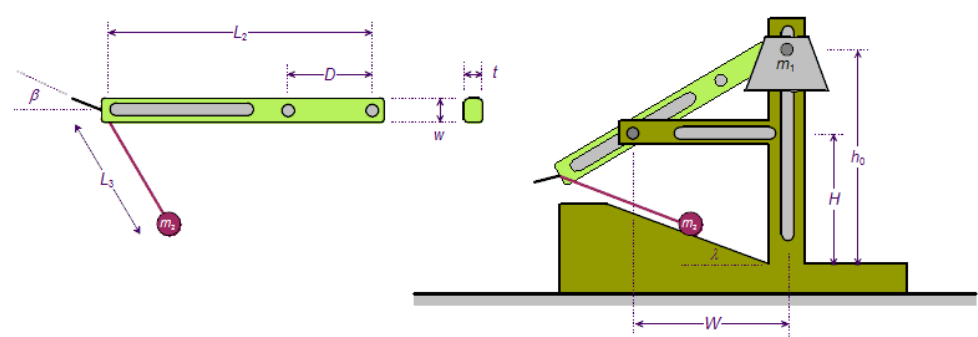

Figura 3 Dimensiones del trebuquete (Eric Constans and Rowan University)

El número de factores de diseño que sí pueden modificarse queda bastante reducido, en este caso a cinco factores, por lo que no va a solicitarse que los alumnos escojan aquellos que puedan pensar que son los más importantes. Se analizarán todos. Dado que el diseño factorial completo tiene un tamaño considerable, esto debe forzar a la elección de un diseño más adecuado. De los cinco factores escogidos, se tienen dos factores de diseño, la longitud del brazo lanzador, L2, y el ángulo que forma el perno de enganche con la dirección principal del brazo, $\beta$, que está relacionado con la dirección de liberación del proyectil. En cuanto a los otros tres factores utilizados son: la masa del proyectil a lanzar, M2, la masa del contrapeso, M1, y la longitud de la honda, L3, si es que se desea lanzar el proyectil con ella. Los valores que pueden tomar estos factores, dado su tamaño de "sobremesa", se encuentran en la Tabla 2.

Tabla 2 Valores razonables para los factores de diseño y uso

\begin{tabular}{|l|l|l|}
\hline Factor & Valor inferior & Valor superior \\
\hline L2 & $0,40 \mathrm{~m}$ & $0,60 \mathrm{~m}$ \\
\hline$\beta$ & -80 o & -20 o \\
\hline $\mathrm{L} 3$ & $0 \mathrm{~m}$ & $0,30 \mathrm{~m}$ \\
\hline $\mathrm{M} 1$ & $20 \mathrm{~kg}$ & $80 \mathrm{~kg}$ \\
\hline $\mathrm{M} 2$ & $0,01 \mathrm{~kg}$ & $0,20 \mathrm{~kg}$ \\
\hline
\end{tabular}

Los cálculos de la distancia recorrida por el proyectil se realizan tomando como base el calculador implementado en la página WEB THE TREBUCHET, y más específicamente las calculadoras de la trebuchet de brazo flotante (Floating Arm), y la opción $4 D O F$ si el trebuquete se lanza sin honda, y $5 D O F$ si se utiliza una honda.

El trabajo a realizar por el equipo está dividido en tres pasos. En el primer paso se hace una toma de contacto con la catapulta y con el simulador utilizado, realizando varios lanzamientos de prueba para familiarizarse con los parámetros de la catapulta y con las diferentes variables respuesta ofrecidas, obteniendo así un conocimiento básico sobre ella. En el segundo paso los estudiantes realizan el análisis del problema, que llevará al planteamiento y ejecución del trabajo con el análisis completo del problema. En el tercer paso los alumnos redactarán una memoria de toda la actividad que han realizado, para terminar preparando dos 
presentaciones, una dirigida a sus compañeros de clase, y otra con un toque más "profesional" que sería el entregado a la hipotética empresa que ha requerido sus servicios. Con algo más de detalle:

1. El primer paso por parte de los alumnos es familiarizarse con la catapulta. Existen muchos diseños de catapulta, y tal vez el escogido (brazo flotante) no coincida con su concepto habitual de catapulta. Después de conocer su aspecto y funcionamiento a través de un vídeo, es necesario que conozcan el simulador, con sus diferentes entradas para seleccionar los parámetros de diseño. Se les invita a que escojan valores y realicen varios lanzamientos, observando la animación ofrecida por el programa. A partir de los pasos dados para "realizar" los lanzamientos, los miembros del grupo se organizan de una forma elemental para poder lidiar con todos los problemas que aparecen en la ejecución de las pruebas.

2. Después de un tiempo razonable, se solicita que los alumnos escojan el diseño que van a utilizar para realizar el análisis. Como ya se ha mencionado, se trata de un modelo de sobremesa en madera, por lo que ciertas dimensiones se fijan según la escala del trebuquete, como ya se ha justificado. Los parámetros de diseño que restan no son tan numerosos como para necesitar de un estudio previo, pero queda en manos de los alumnos su realización, que dependerá del diseño que finalmente escogerán. Los alumnos planifican el experimento, organizan su ejecución, realizan los correspondientes lanzamientos, y analizan la naturaleza de la relación de los factores seleccionados, tanto con el promedio de la distancia recorrida por el proyectil como con su varianza. Una vez analizados los datos y determinados los modelos, se solicitan las dimensiones óptimas de la catapulta para determinadas distancias y masas de proyectiles. Para comprobar que todo está bien, realizan un experimento confirmatorio final en las condiciones propuestas por ellos como óptimas.

3. Realizado el análisis, los alumnos pasan a documentar en una memoria final escrita todo el trabajo realizado, los experimentos, los resultados y las conclusiones obtenidas de su análisis. El enfoque que deben dar a la redacción de este documento es el de un trabajo académico o de investigación.

4. Adicionalmente, los alumnos preparan dos exposiciones orales. En la primera, que dura 18 minutos, explican el documento entregado, prestando especial atención en el diseño escogido y en las soluciones a los problemas encontrados durante la experimentación, para contrastarlas con las de sus compañeros. Después hay una segunda presentación, de 8 minutos, donde los alumnos exponen sus resultados a los responsables de la empresa que los contrató para realizar el estudio, con las conclusiones y las acciones propuestas sobre el diseño de la nueva línea de catapultas. La presentación que realizará cada equipo será escogida al azar entre las dos existentes, y terminará con un turno de preguntas de 10 minutos con el fin de aclarar las cuestiones necesarias.

\section{Competencias ejercitadas}

Con la nueva experiencia planteada a los alumnos se han trabajado diferentes competencias, competencias que según el PROYECTO INSTITUCIONAL DE LA UPV SOBRE COMPETENCIAS TRANSVERSALES, deben adquirir los alumnos. La primera y más obvia es la competencia CT13 Instrumental especifica, el uso de las herramientas estadísticas e informáticas necesarias para su desempeño profesional. En el diseño del trebuquete los alumnos han seleccionado las herramientas estadísticas más adecuadas: diseño de experimentos, análisis de la varianza, regresión y optimización, integrándolas adecuadamente para conseguir el objetivo propuesto. Además, los alumnos han utilizado, tanto programas ofimáticos para la elaboración del documento final y de las presentaciones, como el software estadístico de análisis (Statgraphics Centurion, a través de Polilabs o instalando el software en sus propios equipos, y el software $G r e t l$ ). Así, esta experiencia de optimización del diseño del trebuquete ha permitido profundizar hasta el nivel de dominio III de dicha competencia transversal. La competencia ha sido evaluada a través de la nota obtenida en la memoria grupal del trabajo, transformada en una de las cuatro categorías posibles (No alcanzado, En desarrollo, Adecuado, Excelente). 
Respecto a otras competencias, la dirección del máster asignó a la asignatura la evaluación de la competencia transversal CTO1 Comprensión e integración, competencia que se ejercita y evidencia en diversas fases del trabajo de los alumnos. Esta evidencia se tiene en los tipos de diseños de experimentos por ellos escogidos, ya que éstos muestran haber entendido en cada caso la necesidad de establecer la relación entre la distancia del proyectil y los factores propuestos, de acuerdo con los niveles y variantes seleccionados. Las evidencias de la comprensión e integración aparecen también en la redacción de la memoria final del trabajo y en su posterior exposición, donde se establecen las diferentes conexiones entre conceptos, las relaciones de causa-efecto, los alumnos expresan sus ideas al respecto y se generan las conclusiones, tal como indica el nivel III de dominio de dicha competencia. Esta competencia se ha evaluado mediante la rúbrica que tiene establecida para ello la UPV (Tabla 3).

Tabla 3. Rúbrica para la evaluación de la CT01 propuesta por la UPV

Nivel de dominio III

Resultado de aprendizaje: identificar las carencias de información y utilizar el conocimiento con un enfoque globalizador.

\begin{tabular}{|c|c|c|c|c|c|}
\hline \multirow{2}{*}{ INDICADORES } & \multicolumn{4}{|c|}{ DESCRIPTORES } & \multirow{2}{*}{$\begin{array}{l}\text { EJEMPLOS DE } \\
\text { POSIBLES EVIDENCIAS }\end{array}$} \\
\hline & D. No alcanzado & C. En desarrollo & B. Bien / adecuado & A. Excelente/ejemplar & \\
\hline $\begin{array}{l}\text { Identifica lagunas de } \\
\text { información o falta de } \\
\text { coherencia en las } \\
\text { argumentaciones }\end{array}$ & $\begin{array}{l}\text { No es consciente de las } \\
\text { lagunas o incoherencias } \\
\text { que presentan } \\
\text { determinado } \\
\text { planteamientos }\end{array}$ & $\begin{array}{l}\text { Identifica solo parte de } \\
\text { las lagunas o } \\
\text { incoherencias que } \\
\text { presentan determinado } \\
\text { planteamientos }\end{array}$ & $\begin{array}{l}\text { Detecta incoherencias e } \\
\text { identifica la información } \\
\text { adicional necesaria para } \\
\text { entender determinados } \\
\text { planteamientos }\end{array}$ & $\begin{array}{c}\text { Soluciona las } \\
\text { incoherencias e incorpora } \\
\text { la información adicional } \\
\text { necesaria para entender } \\
\text { determinados } \\
\text { planteamientos }\end{array}$ & $\begin{array}{l}\text { - Lectura y análisis de textos } \\
\text { complejos. } \\
\text { - Realización de trabajos de de } \\
\text { búsqueda de información. } \\
\text { - Propuesta por parte de los } \\
\text { alumnos de posibles } \\
\text { preguntas de examen } \\
\text { relacionadas con un tema } \\
\text { concreto. }\end{array}$ \\
\hline $\begin{array}{l}\text { Establece } \\
\text { generalizaciones o } \\
\text { relaciones causa- } \\
\text { efecto }\end{array}$ & $\begin{array}{l}\text { Generaliza } \\
\text { impulsivamente, no es } \\
\text { capaz de identificar } \\
\text { relaciones de causalidad }\end{array}$ & $\begin{array}{l}\text { Algunas veces generaliza } \\
\text { incorrectamente, } \\
\text { establece relaciones de } \\
\text { causalidad erróneas }\end{array}$ & $\begin{array}{l}\text { Plantea generalizaciones } \\
\text { acertadas e identifica } \\
\text { correctamente relaciones } \\
\text { causa-efecto }\end{array}$ & $\begin{array}{l}\text { Argumenta } \\
\text { correctamente las } \\
\text { relaciones o } \\
\text { generalizaciones } \\
\text { identificadas }\end{array}$ & $\begin{array}{l}\text { Estudio y análisis de casos que } \\
\text { permitan a los alumnos } \\
\text { generalizar a partir de lo que } \\
\text { ocure en situaciones } \\
\text { concretas. }\end{array}$ \\
\hline $\begin{array}{l}\text { Expresa sus ideas y } \\
\text { genera conclusiones, } \\
\text { partiendo de distintos } \\
\text { datos y sus relaciones }\end{array}$ & $\begin{array}{l}\text { Expone sus ideas como } \\
\text { opiniones, sin apoyarse en } \\
\text { datos objetivos }\end{array}$ & $\begin{array}{l}\text { Se apoya en datos, pero } \\
\text { solo tiene en cuenta los } \\
\text { que apoyan su opinión }\end{array}$ & $\begin{array}{l}\text { Expresa sus ideas y } \\
\text { genera conclusiones, } \\
\text { partiendo de distintos } \\
\text { datos y sus relaciones }\end{array}$ & $\begin{array}{l}\text { Evalúa los datos y sus } \\
\text { relaciones para llegar a } \\
\text { conclusiones inéditas, } \\
\text { generando nuevos } \\
\text { conocimientos }\end{array}$ & $\begin{array}{l}\text { Realización de trabajos } \\
\text { académicos que requieran la } \\
\text { lectura previa de un texto } \\
\text { técrico. como por ejemplo un } \\
\text { Real Decreto, una norma o un } \\
\text { estándar. }\end{array}$ \\
\hline $\begin{array}{l}\text { Muestra una visión } \\
\text { sistémica entre varias } \\
\text { disciplinas y/o áreas de } \\
\text { conocimiento }\end{array}$ & $\begin{array}{l}\text { No consigue integrar } \\
\text { elementos de varias } \\
\text { asignaturas, áreas } \\
\text { perspectivas, etc. en un } \\
\text { mismo planteamiento }\end{array}$ & $\begin{array}{l}\text { Integra algunos } \\
\text { elementos de diferentes } \\
\text { ámbitos en un mismo } \\
\text { planteamiento }\end{array}$ & $\begin{array}{l}\text { Enfoca las situaciones de } \\
\text { una manera sistémica, } \\
\text { superando los planos de } \\
\text { cada área de } \\
\text { conocimiento }\end{array}$ & $\begin{array}{c}\text { Presenta una visión } \\
\text { sistémica de la realidad } \\
\text { de un modo } \\
\text { comprensible para los } \\
\text { demás }\end{array}$ & $\begin{array}{l}\text { Realización de un TFM donde se } \\
\text { integren aspectos relacionados } \\
\text { con diversas disciplinas. }\end{array}$ \\
\hline
\end{tabular}

La nueva actividad ha permitido trabajar, además, otras competencias con los alumnos, de manera que si la ERT considera necesario, pudieran evaluarse. En este sentido, las competencias trabajadas con la actividad de simulación del trebuquete han sido las siguientes: la competencia CT02 Aplicación y pensamiento práctico, competencia CT03 Análisis y resolución de problemas, competencia CT08 Comunicación efectiva (tanto en forma oral como escrita), competencia CT09 Pensamiento crítico y, finalmente, la competencia CT12 Planificación y gestión del tiempo.

\section{Resultados}

Los resultados obtenidos en la materia con el cambio del trabajo habitual de laboratorio al simulador del trebuquete son satisfactorios, de acuerdo con las impresiones manifestadas personalmente por los alumnos. Pese a que todo el proceso fue no presencial, y tuvo que hacerse obligatoriamente a través de la plataforma audiovisual, la realización del experimento de forma virtual mantiene la misma sensación de punto final y culminación de la asignatura obtenida con el trabajo presencial. Sin embargo, y dado que los alumnos no han disfrutado de la experiencia de realizar un montaje con sus propias manos, es posible que su satisfacción no sea tan alta (o real) como en anteriores promociones del máster. 
Pese a todo, hay que valorar de forma cuantitativa los resultados obtenidos, y por ello se ha realizado una doble comparación de la innovación propuesta durante el curso 2020-2021 con la anterior promoción, antes de vernos afectados por la pandemia. La primera comparación se basa en el resultado numérico de la evaluación del trabajo de los alumnos, y la segunda, en los porcentajes de alumnos en cada nivel posible de la competencia transversal evaluada, Comprensión e integración.

Respecto a la evaluación del trabajo de los alumnos, su valor se calcula a partir de las notas obtenidas en las diferentes actividades semanales (NotaPracticas), con un peso del $60 \%$ respecto de la calificación final del alumno, y de la nota de la actividad final (NotaActividad), con el $40 \%$ restante.

NotaPracticas es la nota media de los ejercicios realizados en cada una de las cinco sesiones de prácticas de aula informática, que tienen una duración de dos horas y media cada una. A partir de un enunciado, los alumnos deben escoger la técnica estadística de análisis más adecuada, realizar dicho análisis utilizando el software estadístico previsto, y elaborar un informe con las conclusiones y las recomendaciones de mejora. Un punto débil de estas actividades es precisamente el uso de enunciados, pese a que algunos estén basados en problemas reales, procedentes de las colaboraciones de los profesores de la asignatura en empresas. En una asignatura de diseño de experimentos, resulta más razonable que los alumnos en las diferentes prácticas propongan y realicen el diseño, que el que este aparezca ya en el enunciado. Del resultado de la actividad del trabuquete va a depender el que se cambie enunciados por simuladores en las actividades de prácticas.

NotaActividad es la nota en la realización del trabajo relativo al experimento del trebuquete, obtenida a partir de la memoria entregada, de su presentación, y de la "observación" del proceso de trabajo del equipo.

Desde el punto de vista de la evaluación de la asignatura, la utilización del simulador del trabuquete en un laboratorio virtual ha permitido la realización de un trabajo final en grupo, con todo lo que conlleva, sin que existiera contacto físico entre alumnos. La Tabla 4 muestra el detalle del número de alumnos que ha obtenido las diferentes calificaciones en la materia (Excelente, Notable, Aprobado, No Presentado) en cada uno de los dos cursos considerados, y la Figura 4 muestra estos mismos resultados pero representados gráficamente.

Tabla 4 Resultados de la evaluación en 2019-2020 (presencial) y 2020-2021 (online)

\begin{tabular}{|r|r|r|r|r|r|}
\hline & APROBADO & NOTABLE & EXCELENTE & Total & Nota Media \\
\hline $2019-2020$ & 5 & 14 & 3 & \multirow{2}{*}{22} & 7,69 \\
\cline { 2 - 5 } & $22,73 \%$ & $63,64 \%$ & $13,64 \%$ & & \\
\hline $2020-2021$ & 0 & 23 & 3 & 26 & 8,23 \\
\cline { 2 - 5 } & $0,00 \%$ & $88,46 \%$ & $11,54 \%$ & & \\
\hline
\end{tabular}

No se incluye en el presente documento, pero la primera cuestión ha sido comprobar que la promoción 2019-2020 presentaba una distribución de notas "semejantes" a las de promociones anteriores (desde la 2015-2016 hasta la 2019-2020), para poder ser utilizadas en esta comparación, como así fue.

Como se observa en la Tabla 4, la nota media de los alumnos matriculados en 2019-2020 fue de 7,69 frente a un promedio de 8,23 obtenido con la nueva experiencia del simulador, ambos valores calculados sobre 10. Asumiendo que las diferencias entre las medias de ambas promociones tal vez puedan ser debidas a otras cuestiones ajenas al uso del simulador del trebuquete en el trabajo, se ha planteado un test estadístico de comparación de medias (Test $t$ ) presentado en la Tabla 5 . Con un $95 \%$ de confianza, el promedio de la nota obtenida es significativamente diferente en ambas promociones, tal como indica la prueba de comparación de medias de la Tabla 5, siendo la nota del curso 2020-2021 significativamente superior a la del curso 2019-2020. 
Tabla 5 Comparación del promedio de la nota en 2019-2020 (mean1) y 2020-2021 (mean2)

\section{t test to compare means}

Null hypothesis: mean $1=$ mean2

Alt. hypothesis: mean1 NE mean2

assuming equal variances: $\mathrm{t}=-2,47085 \quad \mathrm{P}$-value $=0,0172449$

Reject the null hypothesis for alpha $=0,05$.

Además, tal como se puede observar tanto en la Tabla de frecuencias presentada en la Tabla 4 como en el Diagrama de barras de la Figura 4, en la actual promoción 2020-2021 no hay alumnos con una calificación de aprobado, se mantiene el número de excelentes y aumenta, en consecuencia, el de notables.

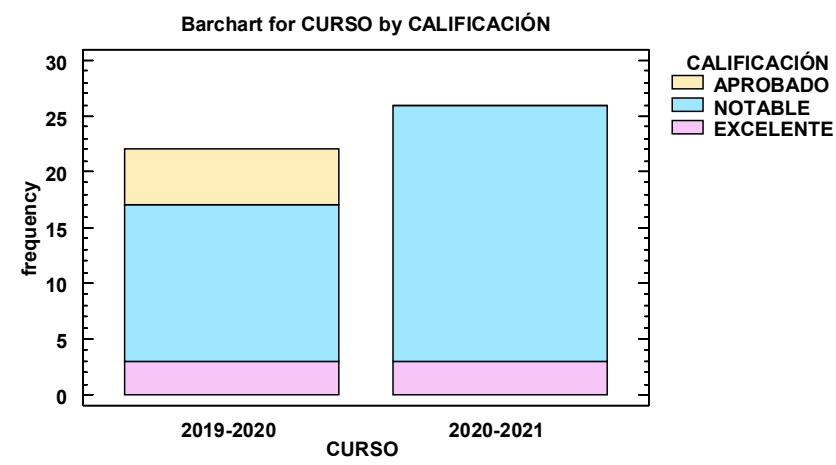

Figura 4 Resultados de la evaluación en 2019-2020 (presencial) 2020-2021 (online)

En lo que respecta a la competencia transversal CT01 Comprensión e integración, los resultados en el presente curso 2020-2021 son también muy positivos, tal como se puede observar en la Tabla de frecuencias recogida en la Tabla 6 y de forma gráfica, en el Diagrama de barras presentado en la Figura 5. Pese a que hay un alumno menos con una calificación de Excelente (A), no hay ninguno en la categoría de En desarrollo (C), y todos los demás se encuentran en la categoría de Adecuado (B). De nuevo, se considera satisfactorio este resultado.

Tabla 6 Resultados de la competencia transversal CT01 en 2019-2020 (presencial) y 2020-2021 (online)

\begin{tabular}{|l|r|r|r|r|}
\hline & A & B & C & Total de fila \\
\hline \multirow{2}{*}{$2019-2020$} & 5 & 12 & 5 & 22 \\
\cline { 2 - 4 } & $22,73 \%$ & $54,55 \%$ & $22,73 \%$ & \\
\cline { 2 - 4 } & $15,38 \%$ & $84,62 \%$ & $0,00 \%$ & 26 \\
\hline Total de columna & 9 & 34 & 5 & 48 \\
\hline
\end{tabular}

Con el fin de determinar si el nivel adquirido en la competencia evaluada CT01 Comprensión e integración es diferente o no en los dos años considerados, se ha realizado una prueba estadística de Independencia cuyo resultado se presenta en la Tabla 7. Dado el P-Valor de 0,0205 (inferior a un 5\%), se deduce, con un 95\% de confianza, que el nivel de competencia alcanzado por los alumnos no es independiente del año de promoción o curso, sino que depende de cuál sea el curso considerado (2019-2020 ó 2020-2021).

Tabla 7 Relación entre los resultados de la competencia transversal CT01 y el curso

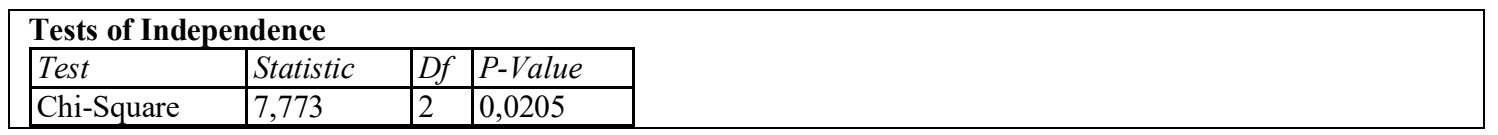




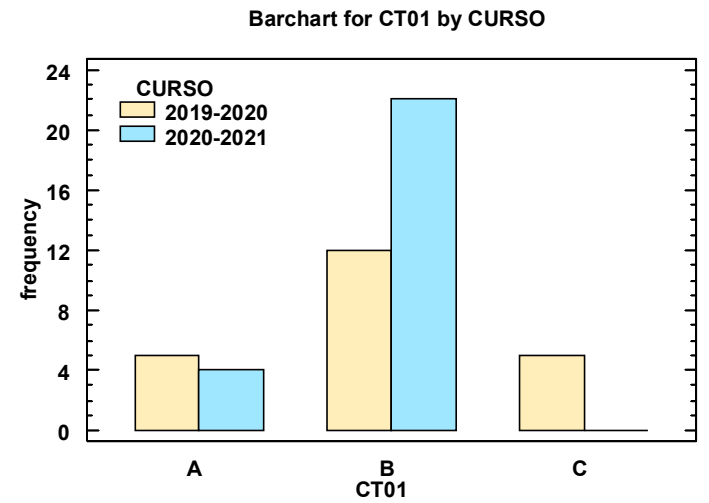

Figura 5 Resultados de la CT01 en 2019-2020 (presencial) 2020-2021 (online)

La conclusión obvia de todo esto es que puede sustituirse de forma satisfactoria la realización de un experimento físico en un aula de laboratorio con un experimento simulado en un laboratorio virtual, sin que se resientan las notas obtenidas ni el nivel de competencia de los alumnos en los aspectos evaluados desde las asignaturas de $D O E$, de carácter marcadamente práctico. Este resultado alienta al uso de simuladores sencillos frente a enunciados estándar en la realización de las prácticas de cada unidad temática.

\section{Conclusiones}

Desde el curso 2015-2016 se llevan a cabo experimentos in situ que simulan procesos complejos en el ámbito de la ingeniería para la evaluación de las asignaturas de DOE del máster de Ingeniería de Análisis de Datos, Mejora de Procesos y Toma de Decisiones de la UPV. Esta participación activa por parte del alumno refuerza los objetivos propuestos en las asignaturas: la selección del diseño de experimentos, la realización del análisis, la interpretación de los resultados y la determinación de las condiciones operativas óptimas asociadas al producto o proceso analizado. En opinión del profesorado de la asignatura, esta metodología, además de estimular y motivar el trabajo, la participación activa del alumno y su aprendizaje, permite desarrollar las competencias de trabajo en equipo, planificación, análisis y resolución de problemas, así como la redacción y presentación pública de informes de carácter técnico-científico.

Las restricciones impuestas por la pandemia de COVID-19, con la imposibilidad del trabajo "codo con codo" de los alumnos ha forzado a la consideración de los laboratorios virtuales como alternativa a la realización de la práctica en el laboratorio real, manteniendo las ventajas que se derivan de la realización de un trabajo aplicado y en grupo.

Una de las ventajas de la incorporación del laboratorio virtual a las asignaturas de $D O E$ es la posibilidad de trabajar de forma síncrona y grupal con alumnos ubicados en diferentes países, en concreto durante el curso 2020-2021, alumnos ubicados en España y en Chile, y alumnos ubicados en diferentes comunidades, por ejemplo durante el presente curso en la Comunidad Valenciana y Castilla León. En este sentido, el laboratorio virtual también puede facilitar el acceso a futuros alumnos con ciertas minusvalías que pudieran impedir, bajo condiciones normales, su acceso físico a las aulas y/o laboratorios o el seguimiento de alguna actividad práctica.

En general y tal como se ha mostrado en la sección 3, los resultados obtenidos con la incorporación del laboratorio virtual a las asignaturas de $D O E$ y, cuantificados tanto en nota del trabajo como en la valoración de la competencia transversal evaluada, Comprensión e integración, deben considerarse positivos. No obstante, desde las asignaturas de $D O E$ nos planteamos si el efecto de la mejora es debido al laboratorio virtual en sí o a otros factores que pudieran estar confundidos, como por ejemplo, que el grupo de alumnos 
tenga un mayor nivel, que exista una motivación extra por parte del alumno debido al interés por la novedad del método, o tal vez por una mayor atención prestada por el profesor dada la situación especial en la que se ha impartido docencia durante el presente curso y el miedo a que algún alumno se quedara descolgado en el camino.

Pese a las ventajas ya mencionadas, creemos conveniente en condiciones normales, no utilizar un laboratorio virtual en la evaluación final de la asignatura. Consideramos que esta evaluación sería más completa y quedaría mejor cubierta con un trabajo "codo con codo" por parte de los alumnos, siendo, además, más abierta, imaginativa y estimulante que cualquier simulación y simplificación de la realidad que se proponga.

Sin embargo, los profesores implicados en esta nueva experiencia consideramos que la introducción de los laboratorios virtuales como alternativa a ciertos ejercicios planteados en las diferentes sesiones de clase puede suponer una mejora de la docencia de dichas sesiones, y en consecuencia, de nuestras asignaturas, con lo que los profesores nos emplazamos en la búsqueda de nuevos simuladores que formen parte del laboratorio virtual DOE. Por ello, nos planteamos también su utilización en otras asignaturas de nuestro departamento que contemplen esta materia en sus programas, como por ejemplo las siguientes: Análisis avanzado de datos en Ingeniería Informática impartida en $4^{\circ}$ curso del Grado de Ingeniería Informática, Ingeniería de la calidad en la industria alimentaria impartida en $3^{\text {er }}$ curso del Grado en Ciencia y Tecnología de los Alimentos, Control y mejora de la calidad en la Ingeniería Química impartida en el $4^{\circ}$ curso del Grado en Ingeniería Química, Técnicas estadísticas avanzadas para el control y la mejora de calidad impartida en el Máster Universitario en Ingeniería Química e Ingeniería de calidad, impartida en el Máster Universitario en Ingeniería Industrial, todas ellas en la UPV.

\section{Referencias}

CHIRIVELLA, V., ALCOVER, R.M. y RICHART, B. (2018). Metodologías activas: simulación de un proceso de centrifugado en Diseño de Experimentos en Ingeniería. En Actas del Congreso Nacional de Innovación Educativa y Docencia en Red 2018, pág 1225-1237, València. http://hdl.handle.net/10251/112867 [Consulta: 23 de marzo de 2021]

Departamento de Estadística e investigación Operativa Aplicadas y Calidad, Máster Universitario en Ingeniería de Análisis de Datos, Mejora de Procesos y Toma de Decisiones

$<$ http://www.upv.es/titulaciones/MUIADMPTD/indexc.html> [Consulta: 23 de marzo de 2021]

Eric Constans and Rowan University, The Trebuchet $<$ http://www.benchtophybrid.com/TB_index.html $>$ [Consulta: 23 de marzo de 2021]

POTKONJAK, V., GARDNER, M., CALLAGHAN, V., MATTILA, P., GUETL, C., PETROVIC, V. M., et al. (2016). "Virtual laboratories for education in science, technology, and engineering: A review". En Computers \& Education, 95, Supplement C, 309-327. DOI: https://doi.org/10.1016/j.compedu.2016.02.002 [Consulta: 23 de marzo de 2021]

ROMERO, R. y ZÚNICA, L.R. (2004). Métodos estadísticos en Ingeniería, Editorial Universitat Politèncica de València.

STAHRE WÄSTBERG, B., ERIKSSON, T., KARLSSON, G. ET AL. "Design considerations for virtual laboratories: A comparative study of two virtual laboratories for learning about gas solubility and colour appearance". Educ Inf Technol 24, 2059-2080 (2019). https://doi.org/10.1007/s10639-018-09857-0 [Consulta: 23 de marzo de 2021]

TORRES, F., ORTIZ, F. G., CANDELAS-HERÍAS, F. A., GIL, P., POMARES, J, ET AL. "El laboratorio virtual como herramienta en el proceso enseñanza-aprendizaje". En II Jornadas de Redes de Investigación en Docencia Universitaria / Alicante : Universidad de Alicante, 2004. http://hdl.handle.net/10045/2274 [Consulta: 23 de marzo de 2021]

Trevor Laughlin, Floating Arm Trebuchet Launch $<$ https://www.youtube.com/watch?v=vYTuzzqdzGc $>$ [Consulta: 23 de marzo de 2021]

Universitat Politècnica de València, Protocolo COVID-19 <https://www.sprl.upv.es/protocolo_covid/01 PROTOCOLO INTERNO ACTUACIÓN UPV V4-5.pdf> [Consulta: 23 de marzo de 2021]

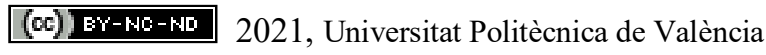

Congreso In-Red (2021) 
Motivando el aprendizaje activo del Diseño de Experimentos en Ingeniería mediante actividades virtuales

Universitat Politècnica de València, Proyecto institucional de las competencias transversales $<$ http://www.upv.es/contenidos/COMPTRAN/> [Consulta: 23 de marzo de 2021].

Wikipedia, Fundibulo <https://es.wikipedia.org/wiki/Fundíbulo $>$ [Consulta: 23 de marzo de 2021]

(c)) BY-NC-ND 2021, Universitat Politècnica de València

Congreso In-Red (2021) 